\title{
Correlation of bronchoalveolar lavage lymphocyte count with the extent of lung fibrosis and with plethysmographic lung volumes in patients with newly recognized hypersensitivity pneumonitis
}

\author{
MONIKA SZTURMOWICZ1, INGA BARAŃSKA², AGNIESZKA SKOCZYLAS \\ MAEGORZATA E. JĘDRYCH ${ }^{1}$, URSZULA DEMKOW \\ ${ }^{1} 1^{\text {st }}$ Department of Lung Diseases, National Tuberculosis and Lung Diseases Research Institute, Warsaw, Poland \\ ${ }^{2}$ Department of Radiology, National Tuberculosis and Lung Diseases Research Institute, Warsaw, Poland \\ ${ }^{3}$ Department of Geriatrics, National Institute of Geriatrics, Rheumatology, and Rehabilitation, Warsaw, Poland \\ ${ }^{4}$ Department of Laboratory Diagnostics and Clinical Immunology of Developmental Age, Medical University of Warsaw, Warsaw, Poland
}

\begin{abstract}
Introduction: Hypersensitivity pneumonitis $(H P)$ is an increasingly recognized interstitial lung disease, presenting with elevated total cell counts and high percentage of lymphocytes in bronchoalveolar lavage fluid $(B A L F)$. Despite many publications, there is no consensus in the literature concerning $B A L F$ cellular composition in patients with prolonged course of $H P$.

Aim: The aim of the present retrospective study was to investigate the influence of disease duration, smoking habits, and the extent of lung fibrosis on BALF cells' population in patients with newly recognized $H P$.

Material and methods: In total, 94 patients (49 females, 45 males), mean age $52( \pm 12)$ years, with $H P$ recognized according to recently proposed criteria, were enrolled into the present study. Chest CT scans were retrospectively reviewed by two independent radiologists. BALF evaluation was performed as a part of routine diagnostics according to recent recommendations.

Results: Percentage of lymphocytes in BALF was significantly lower in patients with lung fibrosis (stage 1 and 2) comparing to those without lung fibrosis (stage 0). Significant correlation was also found between the percentage of BALF lymphocytes and plethysmographic lung volumes, but not with lung transfer capacity for carbon monoxide (TLCO\% pred). Smoking did not influence BALF results in our study group.

Conclusions: BALF lymphocytosis correlated with the presence and the extent of lung fibrosis on chest CT as well as with plethysmographic lung volumes but did not with TLCO and smoking habits in newly recognized HP pneumonitis.
\end{abstract}

Key words: bronchoalveolar lavage, hypersensitivity pneumonitis, high resolution computed tomography, lung fibrosis.

(Cent Eur J Immunol 2020; 45 (3): 276-282)

\section{Introduction}

Hypersensitivity pneumonitis (HP) is an increasingly recognized interstitial lung disease (ILD), usually ranked third, after idiopathic pulmonary fibrosis (IPF) and sarcoidosis or ILD during collagen tissue diseases [1-3]. The first diagnostic step of HP is to identify a symptomatic disease, which develops due to exposition to various, mostly organic, antigens [4]. The second step is to detect an interstitial lung disease with characteristic radiological features in chest computed tomography (chest CT), and the third step involves an evaluation of increased number of lymphocytes in bronchoalveolar lavage fluid (BALF) [4]. Lung biopsy should be considered in case of diagnostic difficulties [5].

According to recent suggestions of experts, two forms of HP have been distinguished: acute type, with symp-

Correspondence: Prof. Monika Szturmowicz, $1^{\text {st }}$ Department of Lung Diseases, National Institute of Tuberculosis and Lung Diseases, 26 Płocka St., 01-138 Warsaw, Poland, e-mail: monika.szturmowicz@gmail.com Submitted: 6.04.2020; Accepted: 30.07.2020 
toms' duration of 6 months or less, and chronic form, with the symptomatic disease lasting for more than 6 months $[4,5]$. Within the group of chronic HPs, two subgroups were described: with or without lung fibrosis [4].

HP presents with increased total cell counts and percentage of lymphocytes in BALF. Lymphocytosis of $60-80 \%$ has been described in acute phase of the disease $[6,7]$. Therefore, pulmonary specialists in DELPHI study indicated BALF lymphocytosis of $40-50 \%$ as a significant factor for differentiation between HP and most other ILDs with lymphocytosis in BALF, such as sarcoidosis, non-specific interstitial pneumonitis, or cryptogenic organizing pneumonia [8].

Despite many published reports, there is no consensus regarding the changes of BALF cellular composition in patients with prolonged HP course. Most authors suggested that BALF lymphocytosis may be lower in chronic fibrosing HP than in acute non-fibrosing disease, especially in smokers [9-12]. Therefore, the value of HP diagnostic algorithm is undefined in chronic phase of the disease.

Therefore, considering the above, we decided to investigate the possible relation between BALF cells' subpopulations and the disease duration, smoking habits, and the extent of lung fibrosis on chest CT in patients with newly recognized HP.

\section{Material and methods}

Clinical presentation, imaging studies, and laboratory testing results of 94 patients, 49 females, 45 males, mean age $52( \pm 12)$ years, with newly recognized HP, were analyzed retrospectively. The applied diagnostic criteria of HP recognition have been described previously $[13,14]$ and consisted of:

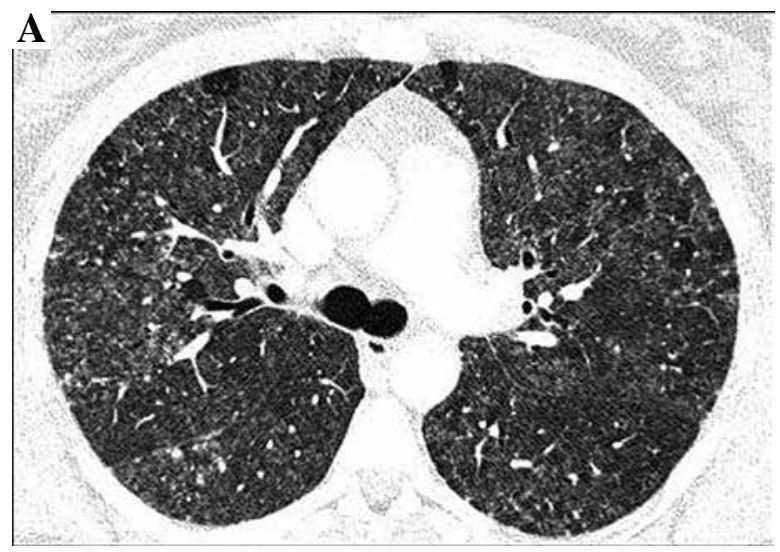

1. Identification of antigen responsible for the symptoms of disease and/or the presence of specific IgG antibodies in serum of symptomatic patients,

2. Characteristic pattern of interstitial lung disease on chest CT (mosaic attenuation pattern of lung parenchyma with air trapping and/or ill-defined centrilobular nodules),

3. Increased percentage of lymphocytes in BALF ( $>30 \%$ in non-smokers and $>20 \%$ in smokers),

4. Histopathology suggestive of HP in the specimen obtained during transbronchial or surgical lung biopsy (in cases not diagnosed with criteria 1-3).

Patients' clinical records were reviewed retrospective$1 y$, and the time from first symptoms of the disease to the diagnosis of HP was determined in months. Acute form of the disease was recognized if the time from first symptoms to diagnosis has not been longer than 6 months, while chronic form, if the symptomatic disease lasted for more than 6 months.

Chest X-rays and chest CT examinations performed at diagnosis, with or without contrast media, on CT scanners with 16 or more rows, were subsequently reviewed by two independent radiologists with more than ten years of experience in ILD diagnostics.

Diagnostic chest CT scan required complete imaging of the lungs on inspiration and slice thickness of $1.5 \mathrm{~mm}$ or less. In some patients, expiration scans have also been performed.

Radiologists evaluated the presence and intensity of typical HP features in CT images, including centrilobular nodules, diffuse ground glass opacifications, air trapping, and mosaic attenuation, and features of lung fibrosis, such as reticulation, traction bronchiectasis, and honeycombing. Lung fibrosis on CT images was staged as: 0. No fibrosis (Fig. 1A), 1. Areas of reticulation with traction bronchiec-

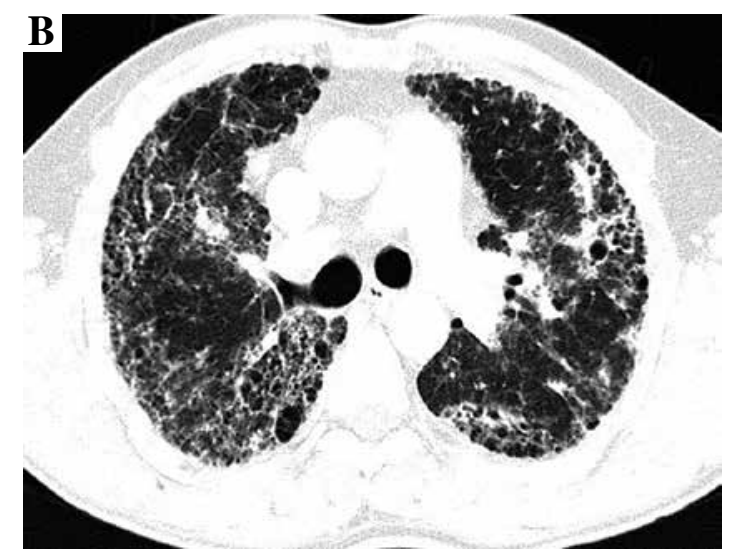

Fig. 1. Non-fibrosing and fibrosing hypersensitivity pneumonitis. Figures present CT axial images of the lung window. A) Bilateral patchy ground-glass opacities, diffuse, poorly defined centrilobular nodules, focal regions of decreased attenuation corresponding to air trapping lobules and normal lungs, characteristic for HP mosaic attenuation, and no features of lung fibrosis (stage 0). B) Fibrosing hypersensitivity pneumonitis - patchy ground-glass opacities and diffuse reticulation with traction bronchiectasis and focal honeycombing, which are the features of advanced lung fibrosis (stage 2) 
Table 1. Characteristics of the study population

\begin{tabular}{|c|c|}
\hline Factor & $n(\%)$ \\
\hline Males & $45(48)$ \\
\hline Females & $49(52)$ \\
\hline Age, years (mean $\pm \mathrm{SD}$ ) & $51.87 \pm 11.73$ \\
\hline Smokers & $39(41)$ \\
\hline Non-smokers & $55(59)$ \\
\hline \multicolumn{2}{|l|}{ Exposure to } \\
\hline Pigeons & $19(20)$ \\
\hline Parrots & $4(4)$ \\
\hline Poultry & $32(34)$ \\
\hline Hay and hay products & $43(46)$ \\
\hline Other & $29(31)$ \\
\hline Not defined & $14(15)$ \\
\hline $\begin{array}{l}\text { Time from first symptoms to diagnosis } \\
\text { (months), median (range) }\end{array}$ & $14(1-360)$ \\
\hline Acute disease & $18(19)$ \\
\hline Chronic disease & $76(81)$ \\
\hline TLC (\% predicted), median (range) & $86(38-146)$ \\
\hline $\mathrm{VC}_{\max }(\%$ predicted), median (range) & $82(31-124)$ \\
\hline $\mathrm{TL}_{\mathrm{CO}}(\%$ predicted $)$, median (range) & $48(10-85)$ \\
\hline \multicolumn{2}{|l|}{ Lung fibrosis on chest $\mathrm{CT}$} \\
\hline Stage 0 & $39(42)$ \\
\hline Stage 1 & $35(37)$ \\
\hline Stage 2 & $20(21)$ \\
\hline
\end{tabular}

$C T$ - computed tomography, TLC-total lung capacity, VC-vital capacity, $T L_{C O}$ - lung transfer capacity for carbon monoxide

tasis, 2. Advanced lung fibrosis with honeycombing and bronchocentric fibrosis (Fig. 1B). Characteristics of study population is presented in Table 1.

BALF was performed as a part of routine testing, according to recent recommendations [15]. To collect BALF, the bronchoscope was placed in the wedge position within the bronchopulmonary segment selected during chest CT examination. Room temperature saline was instilled through a bronchoscope in 10 aliquots, up to maximum of $200 \mathrm{ml}$. The recovered BAL fluid (at least $50 \%$ of infused volume) was filtered through sterile gauze, and centrifuged $\left(4^{\circ} \mathrm{C}, 400 \times \mathrm{g}, 15 \mathrm{~min}\right)$. Cell viability was assessed by trypan blue exclusion. Cytospined smears were stained with May-Grunwald-Giemsa differential staining and assessed by counting of a minimum 600 cells. T cells subpopulations were determined by flow cytometry using FACSCalibur ${ }^{\mathrm{TM}}$ system (Becton Dickinson, San Jose, CA, USA) and Tri TEST CD4/CD8/CD3 monoclonal antibodies (Becton Dickinson, San Jose, CA, USA) [16].

\section{Statistical analysis}

All analyses were performed with $\mathrm{R}$, a software environment for statistical computing and graphics (https:// www.r-project.org/). Continuous variables were presented as medians and ranges, or means and standard deviations, whereas categorical variables were described as percentages of the entire studied population. Distributions' normality and homogeneity of variance of continuous variables in different groups were verified using Shapiro-Wilk test and F-test, respectively. If both criteria were fulfilled, Student's t-test and Mann-Whitney U test were used for comparison of the two groups. Moreover, ANOVA test or Kruskal-Wallis test were applied to compare the three groups. Categorical variables distribution was compared with Pearson's test, with its modifications if applicable. Correlations were analyzed using Spearman's rank order or Pearson's test. Proportions were compared with chisquare test; $p<0.05$ was considered significant.

The project has been approved by Bioethical Committee of National Tuberculosis and Lung Diseases Research Institute (No 14/2019).

\section{Results}

Total BALF cell count was $27.4 \times 10^{6}\left(18.4-42.5 \times 10^{6}\right)$. Median percentage of BALF lymphocytes was $48 \%$ (2.4$84.6 \%$ ). In $82 \%$ of patients, BALF lymphocytosis was higher than $30 \%$. Median of lymphocytes T CD4/CD8 index was 1.9 (0.14-48.4). The results of BALF analysis in relation to the disease duration are shown in Table 2. No significant differences were found between the two groups concerning the percentage of cells in BALF. BALF cells' populations in relation to patients' smoking habits are presented in Table 3.

No significant differences concerning BALF cells' populations between smokers and non-smokers were observed. BALF cells' populations in relation to the presence and the extent of fibrotic changes in chest CT are submitted in Table 4.

The total cell count and relative number of lymphocytes in BALF were significantly lower in patients with lung fibrosis stage 1 and stage 2 comparing to those without lung fibrosis (stage 0) (Table 4, Fig. 2A). Lymphocytosis exceeding $30 \%$ was noticed in all patients with no radiologic signs of lung fibrosis, $77 \%$ of patients with stage 1 lung fibrosis, and $68 \%$ of patients with stage 2 lung fibrosis.

CD4/CD8 index was the highest in case of patients with no signs of lung fibrosis, while the lowest index was observed in patients with advanced lung fibrosis; the differences between the three examined groups were significant (Table 4, Fig. 2B).

The correlations between BALF lymphocytosis and the disease duration, the degree of lung fibrosis as well as plethysmographic lung volumes and $\mathrm{TL}_{\mathrm{CO}}$ are presented in Table 5 . 
Table 2. The results of BALF analysis (medians, ranges) in relation to the disease duration in 94 hypersensitivity pneumonitis (HP) patients

\begin{tabular}{lcccc}
\hline Type of cells & $\begin{array}{c}\text { Whole group } \\
\text { of patients } \\
(\boldsymbol{n}=\mathbf{9 4})\end{array}$ & $\begin{array}{c}\text { Acute HP } \\
\mathbf{6} \text { months } \\
(\boldsymbol{n}=\mathbf{1 8})\end{array}$ & $\begin{array}{c}\text { Chronic HP } \\
>\mathbf{6} \text { months } \\
(\boldsymbol{n}=\mathbf{7 6})\end{array}$ & $\boldsymbol{p}$ (type of test) \\
\hline Total cell count $\times 10^{6}$ & $27.4(3-110)$ & $34(3-87)$ & $27.9(4.3-110)$ & $0.92(\mathrm{MW})$ \\
\hline Lymphocytes $(\%)$ & $48(2.4-84.6)$ & $56.8(14.3-84.6)$ & $47(2.4-84.6)$ & $0.068(\mathrm{TS})$ \\
\hline Lymphocytes & $77(82)$ & $16(88)$ & $52(68)$ & $0.09(\mathrm{CS})$ \\
\hline$>30 \%, n(\%)$ & $1.9(0.14-48.4)$ & $2.6(0.3-7.1)$ & $1.9(0.14-48.4)$ & $0.97(\mathrm{MW})$ \\
\hline CD4/CD8 ratio & $1.1(0-18.9)$ & $0.9(0-5.7)$ & $1.6(0-18.9)$ & $0.26(\mathrm{MW})$ \\
\hline Eosinophils $(\%)$ & $4.05(0-23.5)$ & $4.4(0-16.4)$ & $3.9(0-23.5)$ & $0.41(\mathrm{MW})$ \\
\hline Neutrophils $(\%)$ & $46.85(12.4-91.9)$ & $37.9(14.1-60.4)$ & $47.5(12.4-91.9)$ & $0.09(\mathrm{MW})$ \\
\hline Macrophages $(\%)$ & & &
\end{tabular}

MW-Mann-Whitney U test, TS - Student's t-test, CS - $\chi^{2}$ test

Table 3. Cells populations in BALF (medians, ranges) in relation to smoking habits of 94 hypersensitivity pneumonitis patients

\begin{tabular}{lcccc}
\hline Type of cells & $\begin{array}{c}\text { Whole group } \\
(\boldsymbol{n}=\mathbf{9 4})\end{array}$ & $\begin{array}{c}\text { Smokers } \\
(\boldsymbol{n}=\mathbf{3 9})\end{array}$ & $\begin{array}{c}\text { Non-smokers } \\
(\boldsymbol{n}=\mathbf{5 5})\end{array}$ & $\boldsymbol{p}$ (type of test) \\
\hline Total cell count $\times 10^{6}$ & $27.4(1.8-110)$ & $27.3(3-77)$ & $32.8(1.8-110)$ & $0.36(\mathrm{MW})$ \\
\hline Lymphocytes $(\%)$ & $48(2.4-84.6)$ & $48(7-84.6)$ & $47(4.4-84)$ & $0.93(\mathrm{TS})$ \\
\hline CD4/CD8 ratio & $1.9(0.14-48.4)$ & $2.15(0.14-13.4)$ & $1.56(0.29-48.4)$ & $0.8(\mathrm{MW})$ \\
\hline Eosinophils $(\%)$ & $1.1(0-18.9)$ & $1.1(0-18.9)$ & $1(0-13.3)$ & $0.6(\mathrm{MW})$ \\
\hline Neutrophils $(\%)$ & $4.1(0-23.5)$ & $3.1(0.5-13)$ & $4.3(0-23.5)$ & $0.37(\mathrm{MW})$ \\
\hline Macrophages $(\%)$ & $46.85(12.4-91.9)$ & $47.8(14.1-64.2)$ & $46.7(12.4-91.9)$ & $0.82(\mathrm{MW})$ \\
\hline
\end{tabular}

MW-Mann-Whitney U test, TS - Student's t-test

Table 4. Cells populations in BALF (medians, ranges) in relation to the presence and extent of lung fibrosis in 94 hypersensitivity pneumonitis patients

\begin{tabular}{|c|c|c|c|c|}
\hline Type of cells & $\begin{array}{l}\text { No fibrosis (stage 0) } \\
\qquad(n=39)\end{array}$ & $\begin{array}{l}\text { Fibrosis (stage 1) } \\
\quad(n=35)\end{array}$ & $\begin{array}{c}\text { Fibrosis (stage 2) } \\
\quad(n=20)\end{array}$ & $p$ (type of test) \\
\hline Total cell count $\times 10^{6}$ & $36(3-110)$ & $27.9(1.8-69.2)$ & $21.1(1.9-51)$ & $0.045(\mathrm{KW})$ \\
\hline Lymphocytes (\%) & $62.3(31.3-84.6)$ & $42.7(4.4-74.5)$ & $39.4(2.4-64)$ & 0.00001 (ANOVA) \\
\hline \multicolumn{5}{|l|}{ Lymphocytes, $n(\%)$} \\
\hline$>30 \%$ & $39(100)$ & $27(77)$ & $12(68)$ & $0.04(\mathrm{CS})$ \\
\hline$>40 \%$ & $35(90)$ & $18(51)$ & $9(45)$ & $0.02(\mathrm{CS})$ \\
\hline$>50 \%$ & $28(72)$ & $8(23)$ & $3(15)$ & $0.01(\mathrm{CS})$ \\
\hline $\mathrm{CD} 4 / \mathrm{CD} 8$ ratio & $2.9(0.3-48.4)$ & $1.9(0.14-11.1)$ & $0.65(0.2-7.3)$ & $0.0082(\mathrm{KW})$ \\
\hline Eosinophils (\%) & $0.8(0-9.4)$ & $1.8(0-18.9)$ & $2.2(0.1-13.3)$ & $0.09(\mathrm{KW})$ \\
\hline Neutrophils (\%) & $3.1(0-23.4)$ & $4.3(0.4-22)$ & $4.6(0.4-23.5)$ & $0.19(\mathrm{KW})$ \\
\hline Macrophages (\%) & $33.8(14.1-59.9)$ & $51.2(16.1-91.9)$ & $53.8(33.4-86.7)$ & $0.06(\mathrm{KW})$ \\
\hline
\end{tabular}

KW-Kruskal-Wallis test; ANOVA-ANOVA test, CS - $\chi^{2}$ test 

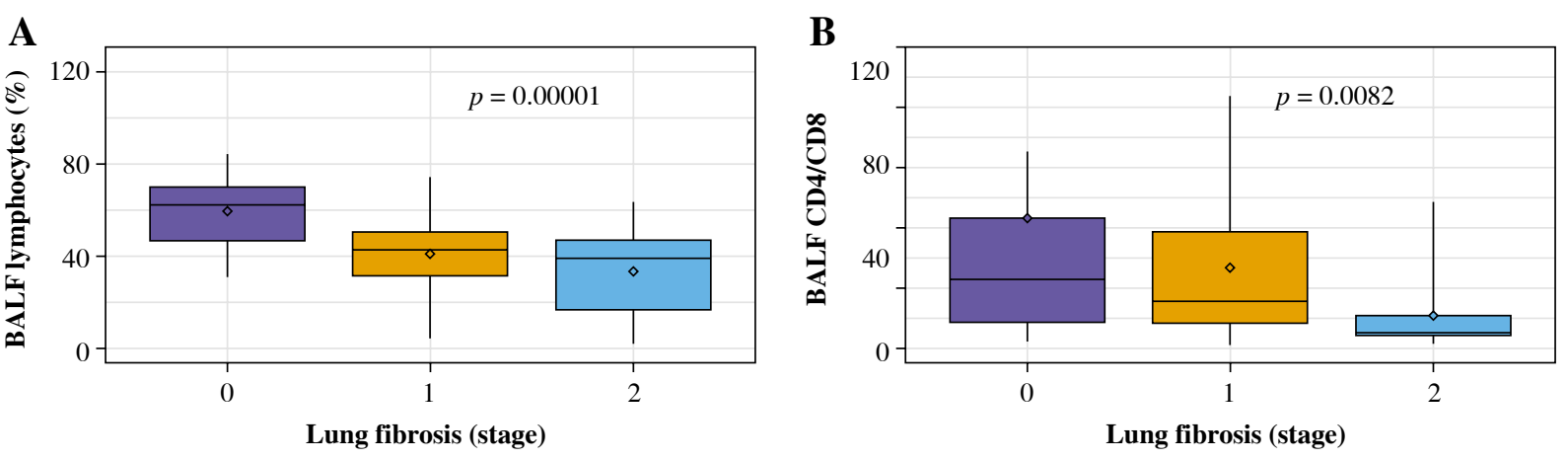

Fig. 2. BALF results in relation to the presence and extent of lung fibrosis in patients with hypersensitivity pneumonitis. A) The percentage of BALF lymphocytes in the groups with no lung fibrosis (stage 0), with lung fibrosis presenting as diffuse reticulation and traction bronchiectasis (stage 1), and with lung fibrosis presenting as diffuse reticulation, traction bronchiectasis, and focal honeycombing (stage 2). B) The proportions of T lymphocytes CD4/CD8 in the groups with no lung fibrosis (stage 0), with lung fibrosis presenting as diffuse reticulation and traction bronchiectasis (stage 1), and with lung fibrosis presenting as diffuse reticulation, traction bronchiectasis, and focal honeycombing (stage 2)

Table 5. Correlations between BALF lymphocytosis (\%) and disease duration, lung volumes on plethysmography, TLCO, and stage of fibrosis on chest CT

\begin{tabular}{lccccc}
\hline Correlation & Disease duration & $\mathbf{T L C} \%$ pred & $\mathbf{V C}_{\mathbf{m a x}}$ \% pred & $\mathbf{T L}_{\mathbf{C o}}$ \% pred & Fibrosis stage 0, 1, 2 \\
\hline BALF lymphocytosis (\%), coefficient & -0.42 & 0.42 & 0.36 & 0.08 & 0.43 \\
\hline$p$-value & 0.0001 & 0.00005 & 0.00046 & 0.41 & 0.0001 \\
\hline Type of test & Spearman & Pearson & Pearson & Pearson & Spearman \\
\hline
\end{tabular}

BALF lymphocyte count was significantly correlated with the stage of lung fibrosis on chest CT as well as the percentage of predicted total lung capacity (TLC) and maximal vital capacity $\left(\mathrm{VC}_{\max }\right)$, but not with lung transfer capacity for carbon monoxide $\left(\mathrm{TL}_{\mathrm{CO}}\right)$. An inverse correlation between disease duration and BALF lymphocyte count was observed.

\section{Discussion}

BALF is an easily performed and well tolerated procedure, with proven diagnostic role in interstitial lung diseases, such as acute and chronic eosinophilic pneumonia, pulmonary alveolar proteinosis, pulmonary Langerhans histiocytosis, and diffuse alveolar hemorrhage [6, 15].

BALF lymphocytosis has been listed as one of important diagnostic criteria of HP, but the results of clinical studies indicate its greatest utility in the acute phase of the disease, and less certain - in chronic HP [17]. Large study conducted in Japan in chronic bird-related and home-related HP, documented lack of increased lymphocyte counts in many cases [18]. Therefore, one of the main issues we addressed in the present study was the relation between the disease duration and BALF cellular composition in patients with newly recognized HP.

Our study group included $19 \%$ of patients with acute HP and $81 \%$ of cases with chronic form of HP. We found significant inverse correlation between BALF lymphocytosis and disease duration. However, the median percentages of BALF lymphocytes in acute HP ( $\leq 6$ months of symptoms duration) was comparable with chronic form of the disease ( $>6$ months of symptoms duration). Such a discrepancy may be explained by relatively high BALF lymphocyte counts in the two forms of HP (values exceeding $30 \%$ were found in $88 \%$ of those with acute HP and $68 \%$ of those with chronic HP). In our opinion, the demonstration of increased percentages of lymphocytes in many patients with prolonged course of HP was the proof of high diagnostic value of BALF examination, even in patients with chronic form of the disease.

Another factor, which potentially may influence BALF composition is cigarettes smoking. According to literature data, an increased total BALF cells' count and alveolar macrophages have been observed in healthy smokers $[6,19]$. In smokers with HP, BALF lymphocyte counts may be lower comparing to non-smokers [20]. Therefore, many authors suggest that BALF lymphocytosis exceeding $20 \%$ should be used as a diagnostic threshold of lymphocytic alveolitis in smoking HP patients, as it corresponds to $95 \%$ of values observed in a healthy smoking population $[7,9]$. Our study group included $41 \%$ of smokers, but we did not observe any differences concerning BALF cells populations between smokers and non-smokers. 
The most important finding of our study group was a significant inverse correlation between the degree of lung fibrosis on chest CT and BALF lymphocytosis $(r=-0.59$, $p=0.0001)$. The more advanced lung fibrosis, the lower median BALF lymphocytosis observed. Therefore, it was not surprising that BALF lymphocytosis was significantly correlated with plethysmographic lung volumes (TLC\% pred and $\mathrm{VC}_{\max } \%$ pred).

BALF lymphocyte counts exceeding $30 \%$ were found in $100 \%$ of patients with no fibrotic lung lesions on chest CT, $77 \%$ of those with stage 1 fibrosis, and $68 \%$ of those with stage 2 fibrosis. If the higher cut-off were used (e.g., $50 \%$ as proposed by DELPHI study), the proportion of patients with elevated BALF lymphocytosis would be $72 \%$, $23 \%$, and $15 \%$, respectively. Therefore, we suggest using a cut-off of $30 \%$ for BALF lymphocytosis when diagnosing HP in patients with fibrotic lung disease. Applying higher cut-off values could markedly decrease the sensitivity of the test. Another possibility suggested by Adams et al. was to increase the diagnostic value of BALF in HP and combining it with trans-bronchial lung biopsy, especially in cases with fibrotic HP, where a lower lymphocyte counts are expected [21].

When the diagnosis of usual interstitial pneumonia (UIP) is considered as alternative to fibrotic HP, the used threshold of BALF lymphocytosis may be even lower than $30 \%$, due to normal BALF lymphocytic count in most UIP patients [22]. Tsilas et al. found BALF lymphocytosis exceeding $20 \%$ in 35 out of 95 patients with non-classified fibrosing lung diseases diagnosed as indeterminate UIP, and one third of them have been subsequently classified as chronic fibrosing HP [23].

In our study, median CD4/CD8 index was 1.9. According to some literature, lymphocytosis in BALF with CD4/CD8 index lower than 1 should be suggestive for HP $[24,25]$. However, other authors questioned the diagnostic importance of CD4/CD8 in HP [26, 27]. Caillaud et al. found CD4/CD8 $<1$ in 30\% of HP patients only [27].

Vasakova et al. speculated that proportion of CD4/CD8 in BALF of HP patients may depend on type of antigen and intensity of exposition as well as the time span between the exposition and BALF collection [20]. In recently described Portuguese cohort of HP patients, CD4/CD8 ratio was significantly lower in patients with acute HP comparing to the remaining ones [28]. It is possible that an increase of CD8 count, which was observed after an acute exposition to antigen has a protective role against lung fibrosis $[20,26]$. The relative shift in balance to $\mathrm{CD} 4$, due to decrease of CD8 count in chronic HP, may favor pro-fibrotic mechanisms [26]. We did not find the differences of CD4/CD8 ratio between acute and chronic form of HP, but surprisingly, we found significantly lower CD4/CD8 ratio in patients with lung fibrosis comparing to those without fibrotic lung disease. The clinical value of this observation is uncertain, due to small sample size, retrospective character of the study, and discrepancies in CD4/CD8 ratio observed in the study group. Therefore, a prospective evaluation performed in larger group of HP patients is required to assess the clinical role of CD4/CD8 ratio as a possible marker of fibrotic lung disease in HP patients.

\section{Conclusions}

In our study group of newly recognized HP patients, BALF lymphocytes' count correlated with the extent of lung fibrosis and plethysmographic lung volumes, but it was not associated with $\mathrm{TL}_{\mathrm{CO}}$ and smoking. The inverse correlation was found between the duration of symptomatic disease and BALF lymphocyte count. The role of CD4/ CD8 index measurement as a marker of fibrotic lung disease requires further investigation.

\section{The authors declare no conflict of interest.}

\section{References}

1. Cottin V, Hirani NA, Hotchkin DL, et al. (2018): Presentation, diagnosis and clinical course of the spectrum of progressive-fibrosing interstitial lung diseases. Eur Respir Rev 27: 180076.

2. Pedraza-Serrano F, Jimenez-Garcia R, Lopez-de-Andres A, et al. (2019): Characteristics and outcomes of patients hospitalized with interstitial lung diseases in Spain, 2014 to 2015. Medicine 98: 21(e15779).

3. Fisher JH, Kolb M, Algamdi M, et al. (2019): Baseline characteristics and comorbidities in the Canadian Registry for pulmonary fibrosis. BMC Pulm Med 19: 223.

4. Vasakova M, Morell F, Walsh S, Leslie K, Raghu G (2017): Hypersensitivity pneumonitis: perspectives in diagnosis and management. Am J Respir Crit Care Med 196: 680-689.

5. Tam WS, Islam T, Nambiar AM (2019): Hypersensitivity pneumonitis (including environmental assessment): diagnosis and management. Curr Pulmonol Rep 8: 131-138.

6. Kebbe J, Abdo T (2017): Interstitial lung disease: the diagnostic role of bronchoscopy. J Thorac Dis 9 (Suppl 10): 996-1010.

7. Salisbury ML, Myers JL, Belloli EA, et al. (2017): Diagnosis and treatment of fibrotic hypersensitivity pneumonia. Where we stand and where we need to go. Am J Respir Crit Care Med 196: 690-699.

8. Morisset J, Johannson KA, Jones KD, et al. (2018): Identification of diagnostic criteria for chronic hypersensitivity pneumonitis. An International Modified Delphi Survey. Am J Respir Crit Care Med 197: 1036-1044.

9. Lacasse Y, Girard M, Cormier Y (2012): Recent advances in hypersensitivity pneumonitis. Chest 142: 208-217.

10. Chiba S, Tsuchiya K, Akashi T, et al. (2016): Chronic hypersensitivity pneumonitis with a usual interstitial pneumonia-like pattern. Correlation between histopathologic and clinical findings. Chest 149: 1473-1481.

11. Takei R, Yamano Y, Kataoka K, et al. (2020): Usefulness of new diagnostic criteria for chronic hypersensitivity pneumonitis established on the basis of a Delphi survey: a Japanese cohort study. Respir Investig 58: 52-58. 
12. Jędrych ME, Szturmowicz M, Bestry I, Kuś J (2016): Hypersensitivity pneumonitis: diagnostic criteria, treatment, prognosis and prevention. Med Pr 67: 517-527.

13. Szturmowicz M, Barańska I, Jędrych ME, et al. (2019): Hypersensitivity pneumonitis recognised in a single pulmonary unit, between 2005 and 2015: comparison with recently proposed diagnostic criteria. Adv Respir Med 87: 83-89.

14. Szturmowicz M, Garczewska B, Jędrych ME, et al. (2019): The value of serum precipitins against specific antigens in patients diagnosed with hypersensitivity pneumonitis - retrospective study. Central Eur J Immunol 44: 390-394.

15. Meyer KC, Raghu G, Baughman RP, et al. (2012): An official American Thoracic Society Clinical Practice Guideline: the clinical utility of bronchoalveolar lavage cellular analysis in interstitial lung disease. Am J Respir Crit Care Med 185: 1004-1014.

16. Szpechciński A, Kopiński P, Giedronowicz D, et al. (2011): Simple flow cytometric protocol of CD4/CD8 lymphocyte ratio assessment in bronchoalveolar lavage fluids from patients with interstitial lung diseases. Ann Quant Cytol Histol 33: 289-296.

17. Morell F, Villar A, Ojanguren I, et al. (2016): Hypersensitivity pneumonitis: challenges in diagnosis and management, avoiding surgical lung biopsy. Semin Respir Crit Care Med 37: 395-405.

18. Okamoto T, Miyazaki Y, Ogura T, et al. (2013): Nationwide epidemiological survey of chronić hypersensitivity pneumonitis in Japan. Respir Investig 51: 191-199.

19. Domagała-Kulawik J, Skirecki T, Maskey-Warzechowska M, et al. (2012): Bronchoalveolar lavage total cell count in interstitial lung diseases - does it matter? Inflammation 35: 803-809.

20. Vasakova M, Selman M, Morell F, et al. (2019): Hypersensitivity pneumonitis: current concepts of pathogenesis and potential targets for treatment. Am J Respir Crit Care Med 200: 301-308.

21. Adams TN, Newton CA, Batra K, et al. (2018): Utility of bronchoalveolar lavage and transbronchial biopsy in patients with hypersensitivity pneumonitis. Lung 196: 617-622.

22. Salisbury ML, Gu T, Murray S, Gross BH, et al. (2019): Hypersensitivity pneumonitis. Radiologic phenotypes are associated with distinct survival time and pulmonary function trajectory. Chest 155: 699-711.

23. Tsilas, Tzouvelekis A, Bouros E et al. (2019): Diagnostic value of BAL lymphocytosis in patients with indeterminate for usual interstitial pneumonia imaging pattern. Eur Respir J 54: 1901144.

24. Ando M, Konishi K, Yoneda R, Tamura M (1991): Difference in the phenotypes of brochoalveolar lavage lymphocytes in patients with summer-type hypersensitivity pneumonitis, farmer's lung, ventilation pneumonitis, and bird fancier's lung: report of a nationwide epidemiologic study in Japan. J Allerg Clin Immunol 87: 1002-1009.

25. Patel A, Ryu J, Reed CJ (2001): Hypersensitivity pneumonitis: current concepts and future questions. J Allerg Clin Immunol 108: 661-870.

26. Murayama J, Yoshizawa Y, Ohtsuka M, Hasegawa S (1993): Lung fibrosis in hypersensitivity pneumonitis. Association with CD4+ but not CD8+ cell dominant alveolitis and insidious onset. Chest 104: 38-43.

27. Caillaud DM, Vergnon JM, Madroszyk A, et al. (2012): Bronchoalveolar lavage in hypersensitivity pneumonitis: a series of 139 patients. Inflamm Allerg Drug Target 11: 15-19.
28. Santos V, Martins N, Sousa C, et al. (2020): Hypersensitivity pneumonitis: main features characterization in a Portuguese cohort. Pulmonology 26: 130-137. 